\title{
Erratum to: Planar Beurling Transform and Bergman Type Spaces
}

\section{Luís V. Pessoa}

Published online: 2 October 2014

(C) Springer Basel 2014

\section{Erratum to: Complex Anal. Oper. Theory (2014) 8:359-381 DOI 10.1007/s11785-012-0268-0}

We proceed with the necessary modifications in the formulation of the second lines of (2.8), (2.9) and (2.11), (2.12) of Theorem 2.1 and Corollary 2.2, respectively, included in the original article.

The second lines of (2.8) and (2.9) of Theorem 2.1 of the original article should be replaced by (1.2) and (1.4) below, respectively. For the reader's convenience, we proceed with one proof of the later assertion.

Theorem 1.1 Let $j \in \mathbb{Z}_{+}$and let $k \in \mathbb{Z}_{ \pm}$. The operators

$$
\begin{aligned}
& \left(S_{\mathbb{D}}\right)^{j}: \mathcal{A}_{(k)}^{2}(\mathbb{D}) \ominus N_{(k), j} \rightarrow \mathcal{A}_{(k+j)}^{2}(\mathbb{D}), \quad k>0 \\
& \left(S_{\mathbb{D}}\right)^{j}: \mathcal{A}_{(k)}^{2}(\mathbb{D}) \rightarrow \mathcal{A}_{(k+j)}^{2}(\mathbb{D}) \ominus N_{j,(-k-j)}, \quad 0<j<-k
\end{aligned}
$$

as well as the following ones

$$
\begin{aligned}
& \left(S_{\mathbb{D}}^{*}\right)^{j}: \mathcal{A}_{(k)}^{2}(\mathbb{D}) \ominus N_{j,(-k)} \rightarrow \mathcal{A}_{(k-j)}^{2}(\mathbb{D}), \quad k<0 \\
& \left(S_{\mathbb{D}}^{*}\right)^{j}: \mathcal{A}_{(k)}^{2}(\mathbb{D}) \rightarrow \mathcal{A}_{(k-j)}^{2}(\mathbb{D}) \ominus N_{(k-j), j}, \quad 0<j<k
\end{aligned}
$$

The online version of the original article can be found under doi:10.1007/s11785-012-0268-0.

This work was partially supported by FCT project PEst-OE/MAT/UI4032/2011.

L. V. Pessoa $(\varangle)$

Departamento de Matemática, Instituto Superior Técnico, Universidade Técnica de Lisboa, Av. Rovisco Pais, 1049-001 Lisboa, Portugal e-mail: lpessoa@math.ist.utl.pt 
are isometric isomorphisms. Furthermore

$$
\operatorname{Ker}\left(S_{\mathbb{D}}^{*}\right)^{j}=\mathcal{A}_{j}^{2}(\mathbb{D}) \text { and } \operatorname{Ker}\left(S_{\mathbb{D}}\right)^{j}=\mathcal{A}_{-j}^{2}(\mathbb{D})
$$

Proof To begin with we assume that $j, k=1,2, \ldots$ From the validity of the so-called exact Dzhuraev's formulas over the unit disk (see [1, Theorem 2.3])

$$
B_{\mathbb{D},-j}=I-\left(S_{\mathbb{D}}^{*}\right)^{j}\left(S_{\mathbb{D}}\right)^{j}
$$

we know that $\left(S_{\mathbb{D}}\right)^{j}$ is a partial isometry. Since $B_{\mathbb{D},-j}$ commute with $B_{\mathbb{D},(k)}$, then $\left(S_{\mathbb{D}}\right)^{j} B_{\mathbb{D},(k)}$ also is a partial isometry. Furthermore,

$$
\begin{aligned}
B_{\mathbb{D},(k)}\left(S_{\mathbb{D}}^{*}\right)^{j}\left(S_{\mathbb{D}}\right)^{j} B_{\mathbb{D},(k)} & =B_{\mathbb{D},(k)}\left(I-B_{\mathbb{D},-j}\right) B_{\mathbb{D},(k)} \\
& =B_{\mathbb{D},(k)}-B_{\mathbb{D},(k)} B_{\mathbb{D},-j}
\end{aligned}
$$

In light of (1.7) we know that $\mathcal{A}_{(k)}^{2}(\mathbb{D}) \ominus N_{(k), j}$ coincides with the initial space of $\left(S_{\mathbb{D}}\right)^{j} B_{\mathbb{D},(k)}$. A simple manipulations then shows that

$$
\begin{aligned}
\left(S_{\mathbb{D}}\right)^{j} B_{\mathbb{D},(k)}\left(S_{\mathbb{D}}^{*}\right)^{j} & =\left(S_{\mathbb{D}}\right)^{j}\left(S_{\mathbb{D}}\right)^{k-1} B_{\mathbb{D}}\left(S_{\mathbb{D}}^{*}\right)^{k-1}\left(S_{\mathbb{D}}^{*}\right)^{j} \\
& =\left(S_{\mathbb{D}}\right)^{j+k-1} B_{\mathbb{D}}\left(S_{\mathbb{D}}^{*}\right)^{j+k-1} \\
& =B_{\mathbb{D},(j+k)}
\end{aligned}
$$

Since the final space of a partial isometry $P$ coincides with the image of the projection $P P^{*}$, then the final space of $\left(S_{\mathbb{D}}\right)^{j} B_{\mathbb{D},(k)}$ is given by $\mathcal{A}_{(j+k)}^{2}(\mathbb{D})$ and (1.1) follows straightforwardly. Let now $k$ and $j$ be such that $0<j<-k$. The initial space of the partial isometry $P$ coincides with the image of the projection $P^{*} P$. Since $B_{\mathbb{D},(k)} B_{\mathbb{D},-j}=0$, it follows from (1.7) that

$$
B_{\mathbb{D},(k)}\left(S_{\mathbb{D}}^{*}\right)^{j}\left(S_{\mathbb{D}}\right)^{j} B_{\mathbb{D},(k)}=B_{\mathbb{D},(k)}, 0<j<-k
$$

Hence, in this case we know that the initial space of $\left(S_{\mathbb{D}}\right)^{j} B_{\mathbb{D},(k)}$ is given by $\mathcal{A}_{(k)}^{2}(\mathbb{D})$. Moreover, from the following equalities

$$
\begin{aligned}
\left(S_{\mathbb{D}}\right)^{j} B_{\mathbb{D},(k)}\left(S_{\mathbb{D}}^{*}\right)^{j} & =\left(S_{\mathbb{D}}\right)^{j}\left(S_{\mathbb{D}}^{*}\right)^{-k-1} B_{\mathbb{D},-1}\left(S_{\mathbb{D}}\right)^{-k-1}\left(S_{\mathbb{D}}^{*}\right)^{j} \\
& =\left(I-B_{\mathbb{D}, j}\right)\left(S_{\mathbb{D}}^{*}\right)^{-k-j-1} B_{\mathbb{D},-1}\left(S_{\mathbb{D}}\right)^{-k-j-1}\left(I-B_{\mathbb{D}, j}\right) \\
& =\left(I-B_{\mathbb{D}, j}\right) B_{\mathbb{D},(k+j)}\left(I-B_{\mathbb{D}, j}\right) \\
& =B_{\mathbb{D},(k+j)}-B_{\mathbb{D}, j} B_{\mathbb{D},(k+j)},
\end{aligned}
$$

we know that the final space of $\left(S_{\mathbb{D}}\right)^{j} B_{\mathbb{D},(k)}$ is given by $\mathcal{A}_{(k+j)}^{2}(\mathbb{D}) \ominus N_{j,(-k-j)}$. Therefore, (1.2) follows straightforwardly.

It is clear that (1.5) follows from the Dzhuraev's formulas for the unit disk. We will finish the proof by showing that (1.3) and (1.4) follows from (1.1) together with 
(1.2). Indeed, if $C$ denotes the anti-linear isomorphism of complex conjugation, i.e. $C f=\bar{f}$, for $f \in L^{2}(\mathbb{D})$, then, for positive integers $j$ and $k$, one easily obtains that

$$
C\left(S_{\mathbb{D}}\right)^{j} C=\left(S_{\mathbb{D}}^{*}\right)^{j}, \quad C\left(\mathcal{A}_{(j)}^{2}(\mathbb{D})\right)=\mathcal{A}_{(-j)}^{2}(\mathbb{D}) \text { and } C\left(N_{j,(k)}\right)=N_{(k), j} .
$$

It is now easily seen that (1.3) and (1.4) is a consequence of (1.1) and (1.2), respectively.

The following result is a consequence of Theorem 1.1 and corrects Corollary 2.2 of the original article. Indeed, the second lines of (2.11) and (2.12) of Corollary 2.2 of the original article should be substituted by (1.10) and (1.12) below, respectively. Fortunately, the mentioned imprecisions do not have more consequences in the original article.

Corollary 1.1 Let $j \in \mathbb{Z}_{+}$and let $k \in \mathbb{Z}_{ \pm}$. The operators

$$
\begin{aligned}
& \left(S_{\mathbb{D}}\right)^{j}: \mathcal{A}_{k}^{2}(\mathbb{D}) \ominus N_{k, j} \rightarrow \mathcal{A}_{k+j}^{2}(\mathbb{D}) \ominus \mathcal{A}_{j}^{2}(\mathbb{D}), \quad k>0 \\
& \left(S_{\mathbb{D}}\right)^{j}: \mathcal{A}_{k}^{2}(\mathbb{D}) \ominus \mathcal{A}_{-j}^{2}(\mathbb{D}) \rightarrow \mathcal{A}_{k+j}^{2}(\mathbb{D}) \ominus N_{j,-k-j}, \quad 0<j<-k
\end{aligned}
$$

as well as the following ones

$$
\begin{aligned}
& \left(S_{\mathbb{D}}^{*}\right)^{j}: \mathcal{A}_{k}^{2}(\mathbb{D}) \ominus N_{j,-k} \rightarrow \mathcal{A}_{k-j}^{2}(\mathbb{D}) \ominus \mathcal{A}_{-j}^{2}(\mathbb{D}), \quad k<0 \\
& \left(S_{\mathbb{D}}^{*}\right)^{j}: \mathcal{A}_{k}^{2}(\mathbb{D}) \ominus \mathcal{A}_{j}^{2}(\mathbb{D}) \rightarrow \mathcal{A}_{k-j}^{2}(\mathbb{D}) \ominus N_{k-j, j}, \quad 0<j<k
\end{aligned}
$$

are isometric isomorphisms.

\section{Reference}

1. Karlovich, Yu.I., Pessoa, L.V.: Poly-Bergman projections and orthogonal decompositions of $L^{2}$-spaces over bounded domains. Oper. Theory Adv. Appl. 181, 263-282 (2008) 